\title{
Compte rendu des captures réalisées lors de la formation Européenne à la détermination des abeilles (COST Super-B Project) dans le Parc National des Calanques
}

\begin{abstract}
par Benoît GesLIN'; Nicolas J.VEREeCKEN ${ }^{2}$; Stuart ROBERTS ${ }^{3}$;A. MURAT AYTEKIN ${ }^{4}$; David GeNOUD ${ }^{5}$; Matthieu AUberT ${ }^{5}$; Rosalie C. F. BURDON ${ }^{6}$; Carlos RUIZ ${ }^{7}$;Alessandro FISOGNI ${ }^{8}$; Floriane FLACHER ${ }^{1}$; Marcin GRABOWSKI ${ }^{9}$; Floriane JACQUEMIN $^{10}$; Viktor KHVIR ${ }^{11}$; Georgi KIRKITADZE ${ }^{12}$; Saskia G. T. KLUMPERS ${ }^{13}$; Karmit LEVY ${ }^{14}$; Stephanie MAHER ${ }^{15}$, Zlata MARKOV ${ }^{16}$;Adrien PERRARD ${ }^{17}$; Laura ROQUER ${ }^{18}$; Lise ROPARS' ; Lucie SCHURR ${ }^{1}$;AndriVARNAVA ${ }^{19}$; \& Denis MICHEZ ${ }^{20}$
\end{abstract}

\section{Introduction}

A l'échelle Européenne, le pourtour méditerranéen français représente un haut lieu de la biodiversité des abeilles sauvages. En effet, sur près de 2000 espèces répertoriées par la Liste Rouge UICN des abeilles sauvages d'Europe (NIETO et al., 2014), on estime que plus de 700 espèces seraient présentes sur une zone allant des Pyrénées Atlantiques aux contreforts Alpins. Cependant, ce chiffre ne reste qu'une estimation de la réelle richesse spécifique des abeilles autour du littoral méditerranéen, pour deux raisons principales. La première raison, soulignée par l'étude de NIETO et al. (2014), tient dans le fait que pour plus de $55 \%$ des 2000 espèces répertoriées en Europe, nous ne disposons pas, actuellement, de suffisamment de données pour pouvoir statuer sur l'état réel de leurs populations ou de leur répartition géographique. La seconde raison réside dans le fait qu'à l'échelle locale la plupart des

\footnotetext{
(1)Aix Marseille Univ, Univ Avignon, CNRS, IRD, IMBE, Aix-Marseille Université - Campus Étoile Faculté des Sciences St-Jérôme Case 42I Av Escadrille Normandie Niémen 13397 Marseille Cedex 20, France

(2)AgroecologyLab, Université libre de Bruxelles (ULB), Boulevard du Triomphe CP 264/2, B-I050 Bruxelles, Belgique.

${ }^{(3)}$ School of Agriculture, Policy and Development, University of Reading, Reading, UK

(4) Hacettepe University Faculty of Science Department of Biology 06800 Beytepe/Ankara/Turkey

(5)Observatoire des Abeilles, Arzens, France

(6) Department of Plant Ecology and Genetics, Evolutionary Biology Centre, Uppsala University, Norbyvägen I8D Uppsala, 75236, Sweden

(7)Dpto. Zoología y Antropología Física, Facultad de Veterinaria, Universidad de Murcia, 30100 Murcia, Spain.

(8)Univ. Lille, CNRS, UMR 8198 - Evo-Eco- Paleo, F-59000 Lille, France

(9)Department of Applied Entomology, Faculty of Horticulture, Biotechnology and Landscape

Architecture, Warsaw University of Life Sciences, Nowoursynowska 159, 02-776 Warsaw,

Poland

(10)Université de Liège-Gembloux Agro-Bio Tech, Unité de recherche BIOSE (ingénierie des

bio-systèmes), Axe Biodiversité et Paysage, Passage des Déportés 2, B-5030 Gembloux,

Belgique.

(II)Faculté de biologie, Université d'Etat de Biélorussie, Minsk, Biélorussie

(12)Agricultural University of Georgia, Institute Of Entomology, Kakha Bendukidze University Campus, \# 240 David Aghmashenebeli Alley, Tbilisi, Georgia

(13)Plant Ecology and Phytochemistry, Institute of Biology Leiden, Leiden University, Sylviusweg 72, 2333 BE Leiden, The Netherlands

(14) Department of Entomology The Robert H Smith Faculty of Agriculture, Food and Environment and The Advanced School for

Environmental Studies, The Hebrew University of Jerusalem

(15)Dept. of Biology, Anglia Ruskin University, Cambridge, UK.

(16) University of Novi Sad, Faculty of Science, Department of Biology and Ecology, 21000 Novi Sad, Serbia

(17)Univ Paris Diderot, iEES Paris UMR 7618, CNRS, Sorbonne Université, Sorbonne Paris Cité, 4 Place Jussieu, 75005, Paris, France

(18)CREAF, Universitat Autònoma de Barcelona, 08193 Cerdanyola del Vallès, Spain

(19)Cyprus University of Technology, Department of Agricultural Sciences, Biotechnology and Food Science, 30 Archbishop Street, 3036 Limassol/Cyprus

(20)Université de Mons, Institut de recherche des Biosciences, Laboratoire de Zoologie, place du parc 23, 7000 Mons, Belgique
}

OSMIA $\mathrm{N}^{\circ} 7$ 
gestionnaires ignorent la biodiversité des abeilles qui vivent réellement sur leurs territoires. Ces deux raisons expliquent principalement les manques qui existent dans les bases de données Européennes.

A titre d'exemple, la base de données du Parc national des Calanques comptait en 2016 moins d'une dizaine espèces d'abeilles dont Apis mellifera et Bombus terrestris. Ce simple constat laissant penser que peu d'études avaient alors été réalisées dans cette zone. C'est une des raisons pour laquelle nous avons décidé d'organiser en 2016 une campagne de collecte et de détermination d'abeilles dans le cadre d'une formation des doctorants/postdoc européens travaillant sur les questions liées aux thématiques de pollinisation (programme Européen COST-Super B ci-après Bee Course http://www.superb-project.eu/, figure 1). Notre objectif principal était de réaliser trois journées de collecte au sein du Parc National des Calanques et ainsi d'apporter de nouvelles informations sur la faune locale d'Apoidea Apiformes.

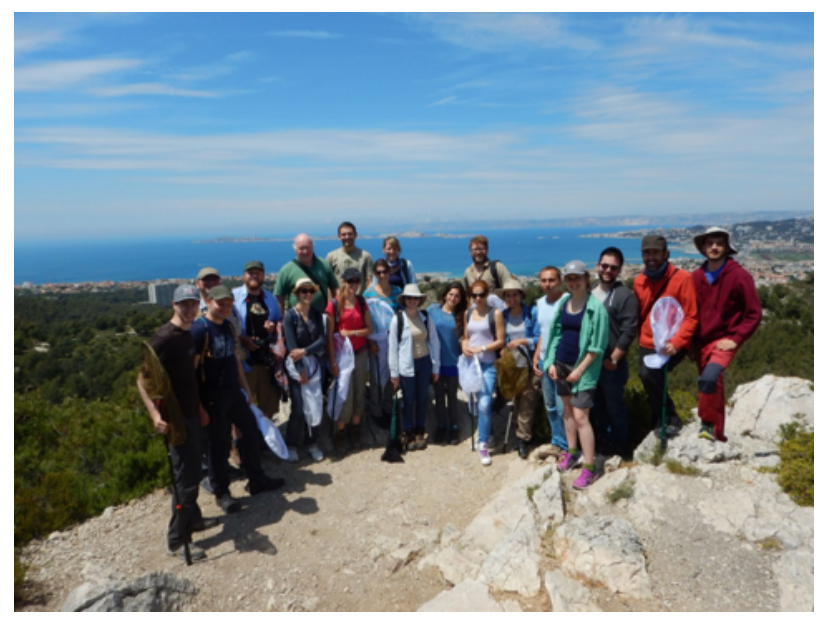

Figure I. Diversité des observateurs du Bee Course 2016. Photographie : N.Vereecken.

Cet article présente les résultats de cette étude de terrain en exposant $1 /$ les différentes espèces capturées, 2/ leur statut de conservation au regard de la liste rouge de l'UICN, et 3/ leurs traits d'histoire de vie.

\section{Méthodes}

Sites

Le Parc National des Calanques (ci-après " PNCal») est le $10^{\mathrm{e}}$ et le plus récent (2012) Parc national français (figure 3 ). Il est le premier parc national périurbain d'Europe. Il s'étend à la fois entre terre et mer, son cœur marin couvrant 435 $\mathrm{km}^{2}$ et son cœur terrestre $85 \mathrm{~km}^{2}$. En terme pédologique, le massif des Calanques est constitué principalement de roches calcaires (ElLILI et al., 2017). Sa flore est bien connue des botanistes (plus de 800 espèces végétales, VÉLA et al., 2001 ; D. Pavon com. pers.). L'habitat majoritaire du PNCal est la garrigue écorchée ou garrigue à cistes composée en majorité de Ciste blanc (Cistus albidus L.), de Ciste de Montpellier (Cistus monspeliensis L.), de Romarin (Rosmarinus officinalis L.) et de Thym (Thymus vulgaris L.) (figure 2). En revanche, la faune d'arthropodes terrestres et notamment d'abeilles sauvages reste très peu documentée. Au sein du PNCal, l'échantillonnage de la Bee Course fut déployé sur trois sites : le campus de Luminy $\left(43^{\circ} 13^{\prime} 52^{\prime \prime} \mathrm{N} ; 05^{\circ} 26^{\prime} 22^{\prime \prime} \mathrm{E}\right)$; la montagne de l'Aigle $\left(43^{\circ} 13{ }^{\prime} 59^{\prime \prime} \mathrm{N} ; 05^{\circ} 23^{\prime} 16^{\prime \prime} \mathrm{E}\right)$ et le vallon du Logisson $\left(43^{\circ} 14^{\prime} 07^{\prime \prime} \mathrm{N} ; 05^{\circ} 30^{\prime} 02^{\prime \prime} \mathrm{E}\right.$ ) (voir figure $3)$.

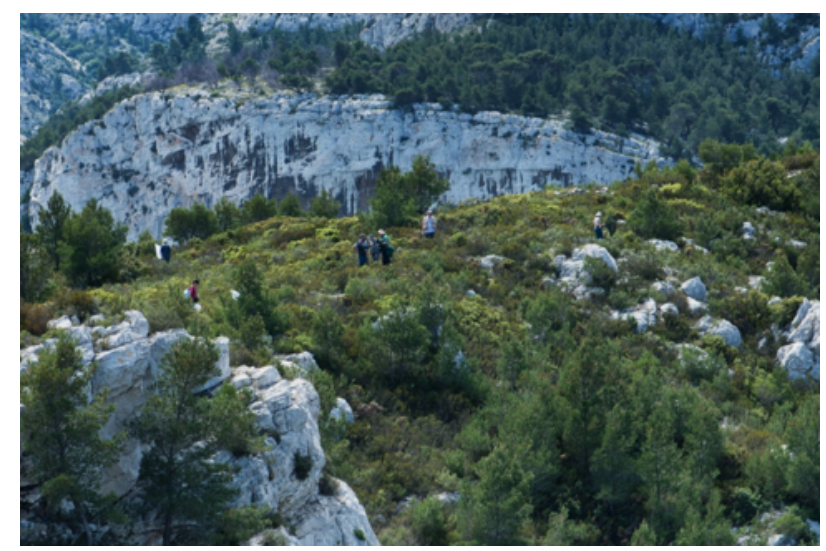

Figure 2. Garrigue écorchée, milieux caractéristique du Parc des Calanques. Photographie : N.Vereecken.

\section{Captures}

Les captures furent réalisées le mardi, mercredi et jeudi 17-18-19 mai 2016. Le temps était alors beau et sec $\left(>15^{\circ} \mathrm{C}\right.$, pas de couverture nuageuse). Ces captures ont été réalisées au filet à papillon ainsi qu'à l'aide d'aspirateurs à bouche selon la méthode de la chasse active par les 20 participants à la formation. Les spécimens ont été étalés le jour même de la capture puis identifiés par des taxonomistes spécialistes de chacun des groupes : Matthieu Aubert (Eucera), Alain Pauly (Halictidae), David Genoud (Andrena), Holger Dathe (Hylaeus), Mickael Kuhlman (Colletes), Michel Terzo (Ceratina), Sebastien Patiny (Panurgus), Pierre Rasmont (Bombus, Melecta et Anthophora) \& Maximilian 


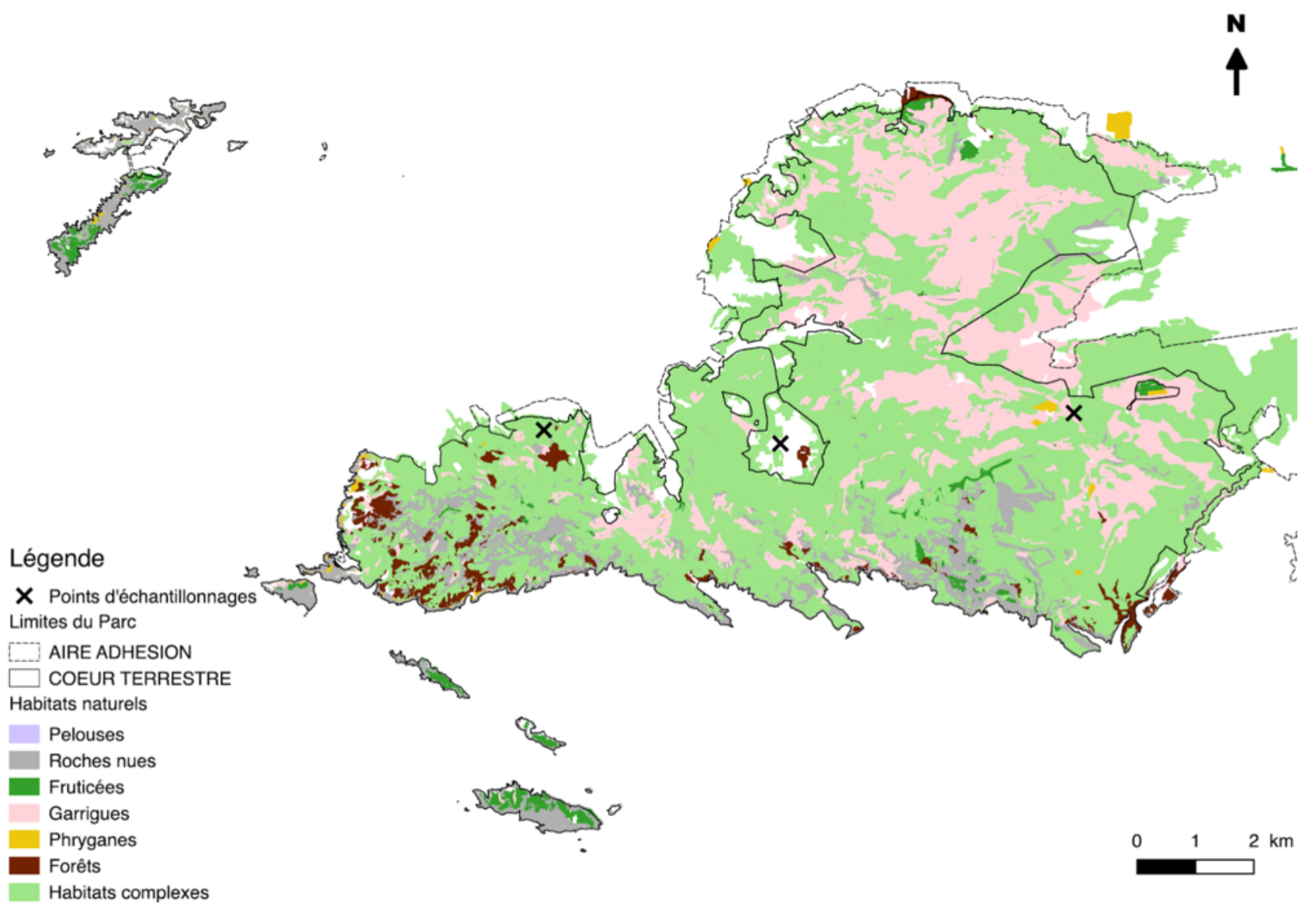

Figure 3. Carte du Parc National des Calanques.

Schwarz et Eric Dufrêne (Nomada). Les traits d'histoire de vie des espèces capturées (lectisme, socialité et substrat de nidification) ont été compilés à partir de la littérature (CHOREIN, 2007 ; CARRÉ, 2008 ; FORTEL, 2014 ; COIFFAITGOMBAUlT et al., 2016 ; GENOUd, 2017 ; MAGHNI et al., 2017) et des bases de données en ligne (de JONG et al., 2014 ; Bees, Wasps \& Ants Recording Society, 2016 ; Atlas Hymenoptera) et par les coauteurs eux-mêmes.

\section{Résultats}

Pour plus de précision sur les données, le lecteur est invité à se référer à l'intégralité de la base de données qui est accessible en ligne sur le site de l'Observatoire des abeilles ici.

\section{Abondance et richesse}

$\mathrm{Au}$ total, 586 individus ont été capturés (figure 4). Ils appartiennent aux familles Andrenidae (105 spécimens), Apidae (75 spécimens), Colletidae (67 spécimens), Halictidae (124 spécimens), Megachilidae (213 spécimens) et Melittidae (2 spécimens - à noter que le genre Dasypoda est classé au sein des Melittidae sensu lato suivant les recommandations de DANFORTH et al., 2013). Le genre le plus représenté est le genre Andrena (79 spécimens), suivi par le genre Hylaeus (60 individus).

Sur ces 586 individus, 103 espèces ont été répertoriées, la plus grande diversité se situant chez les Megachilidae.

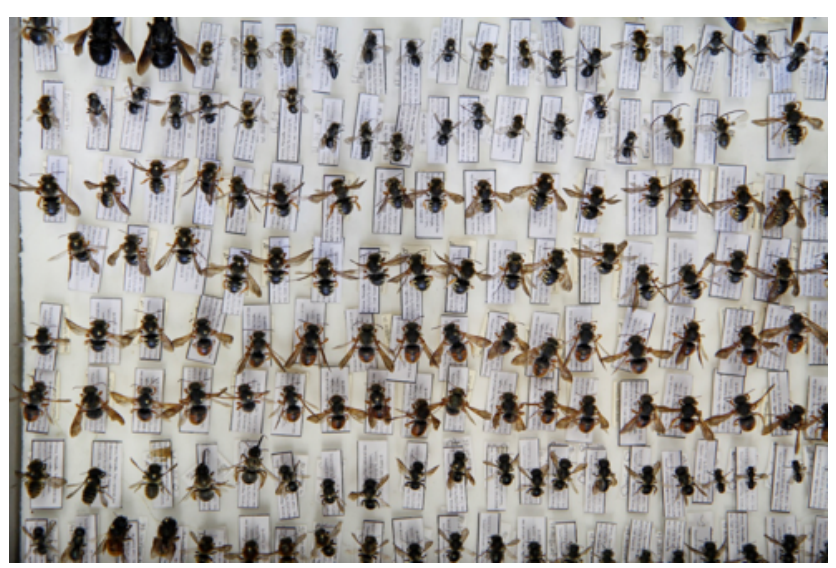

Figure 4. Diversité des spécimens échantillonnés dans le Bee Course 2016. Photographie : L. Schurr. 
Andrenidae (18 espèces) : Andrena agilissima, $A$. brumanensis, A. cinerea, A. combinata, $A$. curtula, A. fabrella, A. flavipes, A. lagopus, $A$. morio, A. nigroaenea, A. niveata, A. ovatula, $A$. pusilla, A. similis, A. simontornyella, $A$. tenuistriata, A. truncatilabris, Panurgus dentites; Apidae (21 espèces): Apis mellifera, Bombus pascuorum, B. ruderatus, B. terrestris, Eucera caspica, E. hispana, E. nigrifacies, Nomada discrepans, N. femoralis, N. glaucopis, $N$. integra, N. rubricoxa, Xylocopa violacea, Anthophora aestivalis, A. femorata, A. mucida, A. plumipes, Melecta albifrons, M. italica, Ceratina chalcites, C. dentiventris.

Colletidae (12 espèces) : Colletes nigricans, Hylaeus brachycephalus, H. clypearis, $H$. euryscapus, H. hyalinatus, H. imparilis, $H$. lineolatus, $H$. pictipes, $H$. pictus, $H$. punctatus, $H$. signatus, $H$. variegates.

Halictidae (23 espèces) : Halictus fulvipes, $H$. maculatus, H. quadricinctus, $H$. scabiosae, $H$. simplex, Lasioglossum albocinctum, L. bimaculatum, L. corvinum, L. glabriusculum, L. griseolum, L. interruptum, L. leucozonium, $L$. malachurum, L. nigripes, L. pauperatum, $L$. pauxillum, L. politum, L. pygmaeum, L. transitorium planulum, L. villosulum, Seladonia gemmea, S. smaragdula sensu lato, Sphecodes ruficrus.

Megachilidae (26 espèces) : Anthidium manicatum, Coelioxys afra, Hoplitis adunca, $H$. anthocopoides, $H$. benoisti, $H$. bisulca, $H$. cristatula, Megachile apicalis, M. melanopyga, M. parietina, M. pilidens, M. willughbiella, Osmia aurulenta, O. brevicornis, O. latreillei, $O$. ligurica, O. melanogaster, O. niveata, $O$. scutellaris, O. signata, O. submicans, $O$. tricornis, O. versicolor, Rhodanthidium septemdentatum, R. sticticum, Stelis signata.

Melittidae (2 espèces) : Dasypoda argentata, Dasypoda cingulata.

\section{Statut de conservation}

Au regard de la Liste Rouge UICN des abeilles européennes (NIETO et al., 2014), quatre espèces d'abeilles considérées comme quasi-menacées (Near-Threatened) ont été capturées : Andrena ovatula, Halictus quadricinctus, Lasioglossum pygmaeum (deux individus), et Dasypoda argentata. Une nouvelle espèce pour la région
PACA a été capturée : Osmia latreillei. Enfin, il est à noter que nous faisons ici mention pour la première fois pour la France de la capture d'un individu de Nomada rubricoxa (SCHWARZ, 1977). Cette femelle a été capturée le 19 mai 2016 sur le site du campus de Luminy. La première mention de cette espèce en France fera l'objet ultérieurement d'une publication indépendante incluant sa description complète.

\section{Traits d'histoire de vie}

En ce qui concerne les préférences alimentaires des espèces identifiées, nous avons répertorié 33 oligolectiques (32\% de la richesse spécifique). Quarante-cinq espèces ont été classées comme polylectiques s.s. (43\%); 12 espèces supplémentaires sont considérées également polylectiques mais avec des préférences particulières pour certaines familles de plantes (voir base de données). Enfin 11 espèces sont non renseignées. L'oligolectisme le plus courant porte sur les Astéracées (14 espèces). La socialité et le grégarisme sont moins représentés parmi nos individus (17 espèces - 16\%) que la nidification solitaire (73 espèces - 70\%, figure 5 ) tandis que nous manquons de précisions pour 9\% d'entre eux. Il est à noter que 51 espèces (49\%) présentent un comportement de nidification terricole, et que $28(27 \%)$ utilisent préférentiellement des cavités pour nidifier (voir la base de données pour plus de précisions). Pour ces dernières, il est intéressant de voir que quelques espèces telles que Osmia aurulenta ou encore Rhodanthidium sticticum sont hélicicoles. Enfin, 26 individus ( $0.5 \%$ de l'abondance totale) appartenant à neuf espèces cleptoparasites ( $8 \%$ de la richesse) ont été identifiés.

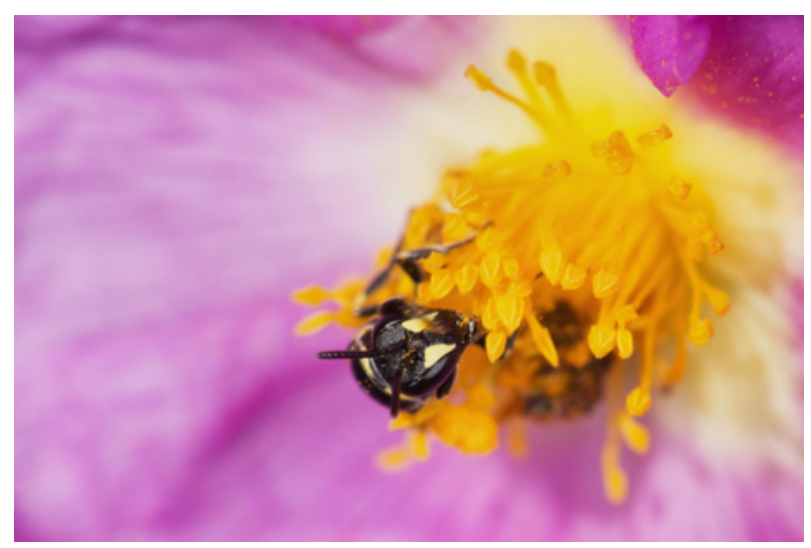

Figure 5. Femelle d'une espèce caulicole (Hylaeus sp.) en train de se nourrir. Photographie : N.Vereecken. 


\section{Discussion}

Un effort d'échantillonnage relativement modéré a permis d'augmenter de manière significative le nombre d'espèces recensées dans le PNCal. Cette étude illustre ainsi les lacunes dans les connaissances relatives à la diversité des abeilles sauvages du Parc. Outre cette étude, peu de données existent à notre connaissance sur le territoire des Calanques en dehors de quelques captures opportunistes ou de collections anciennes. En 2009, au cours de quelques journées de capture au sein du territoire du PNCal, X. Lair avait notamment capturé Lasioglossum soror classée en danger d'extinction ("EN ") par l'UICN, mais ces données n'ont pas été publiées. Des travaux comme ceux réalisés par l'Observatoire des Abeilles dans le Parc National du Mercantour (Inventaire des abeilles sauvages (Hymenoptera : Apoidea : Anthophila) dans le Parc National du Mercantour en 2012, 2013) ou par C. CoiffaitGombault dans le Parc National de Port-Cros (Coiffait-Gombault et al., 2016) sont nécessaires à l'établissement d'une liste d'espèce représentative du PNCal. C'est notamment un des objets de la thèse de doctorat de L. Ropars actuellement en cours.

Ces résultats encourageants ne peuvent former qu'un état des lieux préliminaire à l'évaluation de la diversité des abeilles sauvages dans le PNCal : les captures n'ont été réalisées que sur 3 jours, sur 3 sites, par chasse active d'observateurs plus ou moins expérimentés dans l'échantillonnage, du niveau débutant au niveau le plus chevronné. Or la méthode d'échantillonnage comme la saison de collecte affectent l'observation de la diversité des abeilles (WestPhAL et al., 2008). Nous n'avons donc ici qu'effleuré la diversité réelle des abeilles sauvages dans le PNCal. Les futures campagnes de captures devront être réalisées sur toute la saison de butinage (de février à août et éventuellement en automne), en augmentant le nombre de sites d'échantillonnage et en combinant différentes méthodes de captures (filet + coupelles par exemple).

En seulement trois journées de captures, nous pouvons tout de même noter que la richesse spécifique capturée est tout à fait remarquable puisqu'elle représente plus de $10 \%$ de la faune française d'abeilles sauvages (RASMONT et al., 1995). De plus, cette première campagne de capture présume d'une diversité bien plus importante, notamment au regard de la liste rouge de l'UICN qui évalue la richesse spécifique à près de 700 espèces sur le pourtour méditerranéen français (NIETO et al., 2014). Cependant, l'abondance d'abeilles cleptoparasites semble étonnamment faible aux vues du caractère protégé du PNCal $(0.5 \%$ de l'abondance) et de la publication de Sheffield et ses collaborateurs (SHEFFIELD et al., 2013) qui présentaient ce groupe comme indicateur de la bonne santé d'un écosystème. Ce résultat confirme d'autant plus la nécessité de réaliser de nouvelles investigations dans le PNCal.

L'étude réalisée lors de la Bee Course préfigure ainsi des études à venir et pose les premiers jalons d'une estimation réaliste de la biodiversité d'abeilles sauvages dans le PNCal. Les futures études permettront au $10^{\mathrm{e}}$ Parc national Français de mieux connaître les espèces qui vivent sur son territoire et de renforcer ainsi les mesures de protection envers sa faune sauvage et ses habitats qui doivent faire face à des pressions croissantes.

\section{Remerciements}

Nous tenons à remercier les spécialistes qui ont aidé à déterminer jusqu'au niveau spécifique les spécimens capturés : Mathieu Aubert pour les Eucera et les Megachilidae, Eric Dufrêne et Maximilian Schwarz pour les Nomadinae, David Genoud pour les Andrena, Sébastien Patiny pour les Panurgus, Michael Kuhlmann pour les Colletes, Holger Dathe pour les Hylaeus, Alain Pauly pour les Halictidae, Pierre Rasmont pour les Bombus, Melecta et Anthophora, Michael Terzo pour les Ceratina.

\section{Bibliographie}

ChOREIN A, 2007. Systématique et chorologie des Anthophorini (Hymenoptera: Apidae) de Belgique et du Nord de la France, avec une première analyse de leurs sécrétions volatiles. Mémoire de Licence, 86p.

DE JoNG et al., 2014. Fauna Europaea - all European animal species on the web. Biodivers. Data J. 2, e4034. doi:10.3897/BDJ.2.e4034

GENOUD D, 2017. Synthèse des connaissances sur les hyménoptères apoïdes. Prélèvements 2002 - 2009 et étude 2014. Travaux de la Massane, Tome $\mathrm{n}^{\circ} 108$ 
Illili A, Rabier J, Prudent P, SAlducci MD, HECKENROTH A, LACHAAL M, LAFFONTSchwoB I, 2017. Decision-making criteria for plant-species selection for phytostabilization: Issues of biodiversity and functionality. $J$. Environ. Manage. 201, 215-226

FORTEL L, 2014. Écologie et conservation des abeilles sauvages le long d'un gradient d'urbanisation. Thèse de Doctorat. Université d'Avignon.

CARRÉ G, 2008. Biodiversité, paysages et conservation de la communauté d'abeilles dans les agrosystèmes. Thèse de Doctorat. Université d'Avignon.

Coiffait-Gombault C, Crouzet N, Morisson N, Guilbaud L, VAISSIÈre B, 2016. Diversité des abeilles sauvages (Hymenoptera: Apoidea) de l'île de Porquerolles (France, Var). Sci. Rep. Port-Cros natl. Park 143, 95-143.

MAGHNi N, LOUAI K, ORTIZ-SANCHEZ FJ, RASMONT P, 2017. Les Anthophores de la région des Aurès (nord-est de l'Algérie) (Hymenoptera : Apidae : Anthophorini). Ann. la Soc. Entomol. Fr. 53, 55-73. doi:10.1080/00379271.2017.1305916

NIETO A ET AL., 2015. European Red List of Bees, Luxembourg: Publication Office of the European Union. doi:10.2779/77003

RAsmont P, Ebmer A, Banaszak J, VAN Der ZANDEN G, 1995. Hymenoptera Apoidea Gallica. Liste taxonomique des abeilles de France, de Belgique, de Suisse et du Grand-Duché de Luxembourg, Bull. Soc. Entomol. Fr. 100 (hors série), 1-98

Sheffield CS, Pindar A, Packer L, Kevan PG, 2013. The potential of cleptoparasitic bees as indicator taxa for assessing bee communities. Apidologie 44, 501-510. doi:10.1007/ s13592-013-0200-2

Vela E, Pavon D, Giraud R, Destefano M. \& SAATKAMP A, 2001. Liste des plantes vasculaires du site classé des Calanques (Marseille/Cassis). Bulletin de la Société linnéenne de Provence, 52 : 139-148.

WestPhaL C, et al., 2008. Measuring bee diversity in different European habitats and biogeographical regions. Ecol. Monogr. 78, 653671. doi:10.1890/07-1292.1
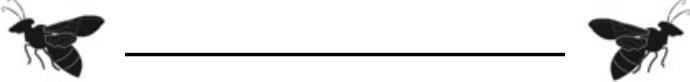